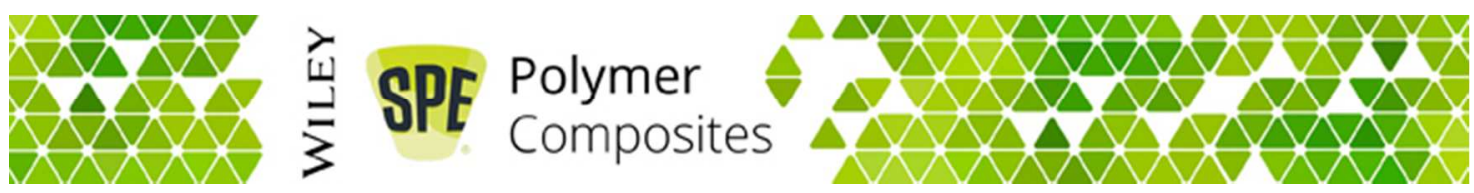

\title{
THERMAL, MECHANICAL AND VISCOELASTIC PROPERTIES OF RECYCLED POLY(ETHYLENE TEREPHTHALATE) (PET) FIBER-REINFORCED UNSATURATED POLYESTER COMPOSITES
}

\begin{tabular}{|r|l|}
\hline Journal: & Polymer Composites \\
\hline Manuscript ID & Draft \\
\hline Wiley - Manuscript type: & Research Article \\
\hline Date Submitted by the Author: & $\mathrm{n} / \mathrm{a}$ \\
\hline Complete List of Authors: & $\begin{array}{l}\text { Dehas, Wided; Universite Ferhat Abbas Setif 1, Génie des Procédés } \\
\text { Guessoum, Melia; Université Ferhat ABBAS Sétif-1, Génie des Procédés } \\
\text { Douibi, Abdelmalek; Universite Ferhat Abbas Setif 1, Génie des Procédés } \\
\text { Jofre-Reche, Jose Antonio; University of Alicante, Adhesion and Adhesives } \\
\text { Laboratory } \\
\text { Martín-Martínez, José Miguel; University of Alicante , Adhesion and } \\
\text { Adhesives Laboratory }\end{array}$ \\
\hline Keywords: & $\begin{array}{l}\text { Unsaturated polyester resin, polyethylene terephthalate (PET) fiber, } \\
\text { composites, mechanical properties, thermal analysis }\end{array}$ \\
\hline \multicolumn{2}{|l}{}
\end{tabular}




\title{
THERMAL, MECHANICAL AND VISCOELASTIC PROPERTIES OF RECYCLED POLY(ETHYLENE TEREPHTHALATE) (PET) FIBER- REINFORCED UNSATURATED POLYESTER COMPOSITES
}

\author{
Wided DEHAS ${ }^{1}$, Melia GUESSOUM ${ }^{1}{ }^{*}$, Abdelmalek DOUIBI ${ }^{2}$, José Antonio JOFRE- \\ RECHE $^{3}$, José Miguel MARTIN-MARTINEZ ${ }^{3}$ \\ ${ }^{1}$ Laboratoire de Physico-Chimie des Hauts Polymères (LPCHP), Département de Génie des \\ Procédés, Faculté de Technologie, Université Ferhat ABBAS Sétif-1, Sétif, Algérie \\ ${ }^{2}$ Laboratoire des matériaux polymériques multiphasiques (LMPMP), Département de Génie des \\ Procédés, Faculté de Technologie, Université Ferhat ABBAS Sétif-1, Sétif, Algérie \\ ${ }^{3}$ Adhesion and Adhesives Laboratory, University of Alicante, 03080 Alicante, Spain
}




\begin{abstract}
Recycled polyethylene terephthalate (PET) fiber has been used as reinforcing filler for unsaturated polyester resin (UPR) in order to obtain UPR/PET fiber composites. Different loadings of PET fibers (5 to $18 \mathrm{phr}$ - parts per hundred parts of resin) of different average lengths (2-3 to $20 \mathrm{~mm}$ ) were added to the UPR. The mechanical properties of the UPR/PET fiber composites increased up to 8 phr PET fiber loading with a length lower than $5 \mathrm{~mm}$, because of the high affinity between the UPR and the PET fiber surface and the good dispersion of the fiber into the matrix. However, higher PET fiber loading caused a decrease in the mechanical properties of the composites because of the agglomeration of the fibers. The UPR/PET fiber composites presented higher storage moduli than the UPR, and an increase of the glass transition temperature in the composites reinforced with 5-8 phr of short PET fiber was found; further, higher degree of crosslinking was reached. The addition of 5-8 phr PET fiber of short length improved the thermal stability of the composites and the post-curing was produced at higher temperature with much reduced enthalpy. The use of 5-8 phr PET fibers with a length lower than $5 \mathrm{~mm}$ was feasible for obtaining tougher UPR composites.
\end{abstract}

Keywords: Unsaturated polyester resin, polyethylene terephthalate (PET) fiber, composites, mechanical properties, thermal analysis, dynamic mechanical thermal analysis, damping factor. 


\section{INTRODUCTION}

The properties of polymeric materials can be improved by adding fillers or fibers. For instance, an increase in the mechanical and thermal properties of reinforced polymers and a decrease of electrical conductivity and gas permeability, have been obtained [1]. Unsaturated polyesters resin (UPR) composites have been widely used in marine, automotive and building industries due to their high chemical resistance, good processability, high mechanical properties and adequate ageing resistance [2,3]. For improving the performance of the UPR composites, different additives, fillers and reinforcements have been proposed [4].

Addition of fibrous reinforcements for improving the properties of thermoset polymers, particularly epoxies and UPR has been widely studied in the literature [5-30]. Among the fiber-reinforced polyester composites, the ones made with E- and S-type glass fibers are the most commonly used [5]. Nevertheless, the lack of affinity between the polymer matrix and the reinforcement engenders a brittle mechanical behavior of the composite because of the inappropriate interfacial interactions in these reinforced thermosets. For obtaining adequate mechanical properties, the fiber-polymer interface must be tailored [6-10] for improving the stresses transfer between the fiber and the polymer matrix. Varga et al. [11] proposed to improve the mechanical properties of glass fiber reinforced polyester composites by treating the fiber surface with polyalkenyl-poly-maleic-anhydride-ester, polyalkenyl-poly-maleicanhydride-amide and polyalkenyl-poly-maleic-anhydride-ester-amide, and they found noticeable increase in the tensile and flexural strength of the glass fiber-treated composites. Rot et al. [12] investigated the influence of the unsaturated polyester resin composition and its styrene content on the interfacial interactions in glass fiber composites. Their results showed that due to their flexibility, the compositions with lower degrees of unsaturation and the higher amount of linear ether glycol presented better adhesion with the glass fibers and as a consequence, the tensile strength of the polyester laminates increased. On the other hand, the fibers surface modification using coupling agents proved efficiency in avoiding interfacial problems, due to the favorable interactions with the polymer matrix via hydrogen or covalent bonds which enhanced the adhesion between them [13]. Organosilanes are the most widely used coupling agents for improving the interfacial adhesion in glass reinforced materials, and their effectiveness depends on the nature and pre-treatment of the fiber, the nature of the silane, the thickness of the silane layer on the fiber surface and the method of impregnation, among others [14-16]. 
Apart from glass fibers, thermosets have also been reinforced with other types of fibers. A thorough investigation of the interfacial shear strength influence on the properties of carbon fiber-reinforced epoxy composites has shown that the shear stresses can be significantly improved when an effective interface is obtained [17]. Fitzer and Weiss [18] have shown that the oxidative surface treatments of carbon fibers resulted in higher interlaminar shear strength of composites with various polymer matrices because of the formation of interfacial chemical bonds. Similarly, Baillie et al. [19] investigated the influence of electrolytic oxidative treatment of carbon fibers on the interfacial shear strength and the fracture toughness of epoxy composites, concluding that the improvement was essentially due to the introduction of acidic functional groups on the surface of the carbon fiber and the existence of chemical bonding.

Currently, there is a great interest in the incorporation of vegetable fibers to UPR for elaborating low density biodegradable bio-composites. Several natural fibers such as flax, sisal, jute, coir, bagasse, alfa and hemp have been studied as reinforcements in UPR composites [20-24]. The surface characteristics of the natural fibers have been widely studied in order to improve their adhesion to polymeric matrices, and different approaches have been used including the chemical or physical modification of the polymer matrix, the fiber or both [25]. Hill and Abdul Khalil [26] investigated the effect of different chemical modifications (acetylation, silane treatment, titanate coupling) on the mechanical properties of coir and oil palm fiber-reinforced polyester composites. A slight increase in tensile strength, modulus, and impact strength of the composites reinforced with modified fibers was obtained. Pothan et al. [27] examined the mechanical properties of various silane treated and mercerized banana fiber-reinforced polyester composites and concluded that the alkali treated fibers rendered composites with high mechanical properties due to the better packing of the cellulose chains after dissolution of the lignin. Rout et al. [28] followed the effect of the alkali treatment of coir on the performance of coir-polyester composites and found that as the concentration of sodium hydroxide increased, the mechanical properties of the composite decreased due to the cell wall thickening that lead to poor adhesion. Moreover, Rokbi et al. [29] observed that the flexural properties of alfa fiber/UPR composites decreased after alkali treatment of the alfa fiber with $5 \mathrm{wt} \%$ of sodium hydroxide $(\mathrm{NaOH})$ for 48 hours, and they ascribed this decrease to the reduction of lignin that bonds the cellulose fibrils together. Herrera-Franco and Valadez-González [30] highlighted the importance of the determination of the critical fiber length for composites processing and they concluded that this parameter can be affected by the fiber treatment because the improved interfacial adhesion was related to the considerable reduction of the aspect ratio. On the other hand, Sreekumar et al. [22] studied the tensile and 
flexural strength of sisal fiber-reinforced polyester composites prepared by resin transfer molding (RTM) and compression molding (CM) as a function of the fiber length and the fiber loading. The study revealed that the tensile strength, Young's modulus, flexural strength and flexural modulus of the RTM processed composites were higher than for the CM ones, and the better properties were found for the composites made with fibers having a length of 30 $\mathrm{mm}$. When the fiber length was increased, a sharp decrease of the mechanical properties was observed due to the poor wetting and dispersion of the fibers into the polymer matrix.

In this paper, poly(ethylene terephthalate) (PET) fibers derived from the recycling of postconsumption PET bottles is proposed as a new type of reinforcement for UPR composites. To the best of our knowledge, no study has been performed on the addition of PET fibers as filler for UPR. Because of the lower density of the PET fibers as compared to other fibers, the aim of this study is valorize the recycled PET fiber for producing low density UPR composites. On the other hand, because of the ester chemical structure of both the PET fiber and the UPR polymer, good interfacial adhesion between them can be anticipated and no need of surface modification should be necessary for obtaining good interfacial interactions. The UPR/PET fiber composites were prepared using different amounts of recycled PET fibers $(5,8,13$ and $18 \mathrm{phr})$ with different lengths $(2-3,5,10,15$ and $20 \mathrm{~mm})$.

\section{EXPERIMENTAL}

\subsection{Materials}

Isophthalic unsaturated polyester resin Norsodyne F44273 (Exxon Mobil Chemical, CCP Composite Cray Valley, France) was used. This resin has a viscosity of $373 \mathrm{mPa} . \mathrm{s}$ at $25^{\circ} \mathrm{C}$. $1.5 \mathrm{phr}$ of methyl ethyl ketone peroxide (MEKP) and $0.25 \mathrm{vol} \%$ of cobalt octoate were used as initiator and accelerator of the polyester resin, respectively. The poly(ethylene terephthalate) (PET) fibers were supplied by RET-PLAST (Mezloug-Setif, Algeria) and they derived from post-consumer PET bottle recycling.

\subsection{Preparation of the UPR/PET fiber composites}

The PET fibers were cut to the average lengths of 2-3, 5, 10, 15 and $20 \mathrm{~mm}$. $227 \mathrm{~g}$ of the unsaturated polyester resin containing $0.25 \mathrm{vol} \%$ cobalt octoate was gently mixed manually by using a wood spatula in a cylindrical metal container (diameter: $10 \mathrm{~cm}$, height: $11 \mathrm{~cm}$ ). Then, 5, 8, 13 and $18 \mathrm{phr}$ of PET fibers of average length of 2-3 mm were added and mixed manually for 5 minutes. Finally, $1.5 \mathrm{phr}$ of MEKP was added for crosslinking of the 
UPR resin. The mixture was placed on a Teflon plate on which a wood mold of dimensions of $(250 \times 250 \times 4) \mathrm{mm}^{3}$ was placed. After ensuring a good distribution of the liquid mixture over the entire surface of the mold and the release of air bubbles, the mixture was allowed to cure at room temperature for 24 hours followed by heating at $40{ }^{\circ} \mathrm{C}$ for 16 hours. Composite plates of $4 \mathrm{~mm}$ thick were obtained. Additional UPR/PET fiber composites were prepared by adding 5 and $8 \mathrm{phr}$ of PET fibers of different lengths $(5,10,15$ and $20 \mathrm{~mm})$. The same procedure described above was used to prepare the composites. Finally a blank composite without PET fibers was prepared as reference by using the same procedure employed to prepare the UPR/PET fiber composites.

\subsection{Experimental techniques}

Density measurement. The density of the composites was measured in Ohaus type AR1140 balance (Ohaus, China) according to the standard ISO 1183, method A, designed for materials having a density greater than $1 \mathrm{~g} / \mathrm{cm}^{3}$. For the calculation of the density, the equation (1) was used:

$$
\mathrm{d}=\frac{\text { Waxp(fl) }}{W_{\mathrm{a}}-W_{2}}+0.0012 \mathrm{~g} / \mathrm{cm}^{2}
$$

Where $\mathrm{d}$ is the sample density in $\mathrm{g} / \mathrm{cm}^{3}, \rho(\mathrm{fl})$ is the density of the immersion liquid (water), Wa is the weight of the sample in air (g), Wfl is the absolute value of the hydrostatic thrust $(\mathrm{g})$, and $0.0012 \mathrm{~g} / \mathrm{cm}^{3}$ is the density of air under normal conditions.

Stress-strain tests. The mechanical properties of the composites were obtained by stress-strain tests of rectangular shaped samples of dimensions $(125 \times 25 \times 4) \mathrm{mm}^{3}$. The stress-strain tests were performed in WDT universal testing machine (WDT, Jingmi Cheng, China) using a pulling rate of $2 \mathrm{~mm} / \mathrm{min}$. The tensile strength and elongation-at-break denoted as or and $\varepsilon$, respectively, were evaluated from the stress-strain curves.

Impact test. Izod impact test of unnotched specimens with dimensions $(65 \times 15 \times 4) \mathrm{mm}^{3}$ was performed according to ISO180 standard using Resil Impactor pendulum apparatus $(7.5 \mathrm{~kg})$ (Ceast, Italy). 
Thermal gravimetric analysis (TGA). Thermal gravimetric analysis of the composites was carried out in Q-500 TA instrument (TA Instruments, New Castle, Delaware, USA). About 15 $\mathrm{mg}$ of the composite was heated under inert atmosphere (nitrogen flow rate: $100 \mathrm{ml} / \mathrm{min}$ ) from room temperature to $600{ }^{\circ} \mathrm{C}$ using a heating rate of $10{ }^{\circ} \mathrm{C} / \mathrm{min}$.

Differential scanning calorimetry (DSC). The thermal and structural properties of the composites were measured by DSC in TA Q-100 apparatus (TA instruments, New Castle, Delaware, USA). 5-10 mg of composite was placed in a sealed aluminum pan. The samples were heated from 25 to $250{ }^{\circ} \mathrm{C}$ using a heating rate of $10^{\circ} \mathrm{C} / \mathrm{min}$, then they were maintained at $250{ }^{\circ} \mathrm{C}$ for 2 minutes followed by cooling down to $25{ }^{\circ} \mathrm{C}$. Afterwards, a second DSC heating run was carried out from 25 up to $250{ }^{\circ} \mathrm{C}$ using a heating rate of $10{ }^{\circ} \mathrm{C} / \mathrm{min}$. All experiments were carried out under nitrogen atmosphere (flow rate: $50 \mathrm{ml} / \mathrm{min}$ ).

Dynamical mechanical thermal analysis (DMTA). The viscoelastic properties of the composites were studied in dynamical mechanical thermal analyzer DMAQ-800TA (TA instruments, New Castle, Delaware, USA). The tests were performed using rectangular samples with dimensions of $(35 \times 10 \times 4) \mathrm{mm}^{3}$ using the single cantilever geometry. The composites were heated from -50 to $180{ }^{\circ} \mathrm{C}$ using a heating rate of $5{ }^{\circ} \mathrm{C} / \mathrm{min}$. The frequency was $1 \mathrm{~Hz}$ and the amplitude was set at $10 \mu \mathrm{m}$. All experiments were carried out under nitrogen atmosphere (flow: $50 \mathrm{ml} / \mathrm{min}$ ).

Scanning electron microscopy (SEM). The topography of the PET fibers surface and the composites was observed by scanning electron microscopy in Hitachi S3000N instrument (Hitachi Ltd., Tokyo, Japan). The analysis of the composites was performed by observation of the fractured surfaces produced by breaking them mechanically once they were frozen by immersion in liquid nitrogen. For improving the contrast, the samples were gold coated in Balzers SCD 004 sputtering unit (Oerlikon Balzers, Liechtenstein).

\section{RESULTS AND DISCUSSION}

The variation of the density of the UPR/PET fiber composites as a function of their fiber content is shown in Figure 1. The low density of the PET fiber does not induce significant change in the density of the UPR resin, and all composites have very close densities to that of 
the matrix. This is an advantage of the UPR/PET fiber composites since their lightness is an additional asset in their performance.

Figure 2 shows the SEM micrograph of the PET fibers. PET fibers have a smooth surface and a hollow cylindrical structure. Their average diameter is $60-70 \mu \mathrm{m}$. Because of the hollow structure of the PET fibers, the low increase in the density of the composites can be anticipated and therefore despite the addition of high PET fiber content, i.e. $18 \mathrm{phr}$, the composite density remains almost constant.

\subsection{Influence of the PET fiber content on the properties of the UPR/PET fiber composites}

Figure 3 a shows the variation of the tensile strength of the UPR/PET fiber composites as a function of the fiber content. A significant increase in tensile strength is found when 5 to 8 phr of PET fiber is added. Addition of higher amounts causes a decrease of the tensile strength of the composites although it is higher than in the neat UPR resin. On the other hand, the addition of 5-8 phr of PET fibers increases also the elongation-at-break of the UPR/PET fiber composites (from $0.94 \%$ for the unfilled resin to $2.78 \%$, for the composite with $8 \mathrm{phr}$ PET fiber). The elongation-at-break decreases slightly when increasing the PET fiber loading (Figure 3b). Therefore, the addition of PET fiber seems impart toughness to the UPR/PET fiber composites causing an increase in both the tensile strength and the elongation-at-break. The toughness imparted to the composites can be ascribed to the compatibility between the UPR and the PET fiber derived from the existence of polar ester groups in the chemical structure of both polymers; this compatibility should favor net interfacial interactions between the PET fiber surface and the UPR matrix.

Figure 4 shows the variation of the Izod impact strength of the UPR/PET fiber composites as a function of the fiber loading. In general, the impact strength of the composites does not vary by adding PET fiber except for the one with $8 \mathrm{phr}$ in which a noticeable increase in resilience is obtained. This trend can be ascribed to the different degree of dispersion of the PET fibers in the UPR matrix and this seems optimal in the composite containing a concentration of 8 phr. 
The dispersion of the PET fibers in the UPR matrix was assessed by SEM. Figure 5 shows the SEM micrographs of the fractured surfaces of UPR/PET fiber composites reinforced with 5, 8 and $18 \mathrm{phr}$ of fibers $(2-3 \mathrm{~mm})$. It is worth noting that all the composites show little evidence of fiber pulled out and, indeed, after fracture the PET fibers are embedded into the matrix and they present a good interfacial adhesion to the UPR resin. The splitting of the fibers results from their ability to withstand the applied load that is transferred from the matrix. This would not be possible if the adhesion of the fiber to the matrix is not sufficiently strong. During mechanical tests, the rupture does not occur at the interface between the fiber and the matrix because the stresses are transferred to the resin. According to Figure 5, the addition of 5-8 phr of PET fibers allows a good dispersion in the UPR matrix and seems that a good wettability of the fibers by the resin is produced. The development of strong interfacial interactions ensures a larger contact surface enabling a better stress transfer between the two phases of the composite [1-4]. Furthermore, the composites containing 5-8 phr of PET fibers show evidences of high resistance to the fracture because of the fiber deformation and the roughness and irregularity of the fractured surface, especially in the composite with $8 \mathrm{phr}$ of PET fibers. However, for the composite with 18 phr of PET fibers, the SEM micrograph of Figure 5 shows agglomerates that reveal poorer impregnation with the resin, and therefore an efficient stress transfer cannot be ensured because the fiber/fiber interactions are favored. Although the interactions UPR/PET fiber are favorable, the defects induced by the proximity of the PET fibers may act as initiators of the brittle fracture in the composite.

The thermal and structural properties of the UPR/PET fiber composites were assessed by DSC. Figure 6a represents the DSC thermogram of the PET fiber which shows the glass transition temperature $\left(\mathrm{T}_{\mathrm{g}}\right)$ at $78{ }^{\circ} \mathrm{C}$, the exothermic crystallization peak around $126{ }^{\circ} \mathrm{C}$, and the endothermic melting peak at about $250^{\circ} \mathrm{C}$.

Figures $6 \mathrm{~b}$ and $6 \mathrm{c}$ show the DSC thermograms corresponding to the first and second heating runs of the neat UPR. During the first heating run, the glass transition of the UPR initiates at $69^{\circ} \mathrm{C}$ but vitrification appears before ending the glass transition due to the starting of postcuring as it seems that it was not completed during processing. The curing of thermoset resins is complex due to the interaction between the chemical kinetics and changes in their physical properties. Gelation and vitrification are the most important changes the material undergoes during curing. Vitrification is a change from the liquid or rubbery state to the glassy state due 
to an increase in both the crosslinking density and the molecular weight of the material during the curing process. This transformation occurs when the $T_{g}$ of the material coincides with the curing temperature. At this moment, curing within the glassy state becomes extremely slow and the reactive process changes from being chemically controlled to being controlled by diffusion [31]. The increase in the temperature during the first DSC heating run re-starts the curing of the UPR resin and an exothermic peak between 90 and $170{ }^{\circ} \mathrm{C}$ is noticed due to post-curing; the maximum temperature of the post-curing is located at $112{ }^{\circ} \mathrm{C}$ and the enthalpy of post-curing is $21 \mathrm{~J} / \mathrm{g}$. The complete curing of the UPR composite during the DSC experiment is noticed by the absence of exothermic peaks in the second DSC heating run in which the Tg of the fully cured UPR composite appears at $91{ }^{\circ} \mathrm{C}$.

Figure 7a shows the DSC thermograms corresponding to the first heating run of the UPR/PET fiber composites. All composites exhibit the glass transition temperature, the vitrification process and the exothermic peak due to post-curing. As compared to the neat UPR, the temperature at which post-cure appears in the composites increases irrespective of the added amount of PET fiber (Table 1). The post-curing enthalpy is notably reduced in the composites loaded with 5-8 phr PET fibers which facilitate the curing of the composites, likely due to the net interfacial interactions between the fibers and the resin. However, the addition of amounts of PET fibers higher than $8 \mathrm{phr}$ has a minor effect as the post-curing enthalpy is somewhat similar to that of the neat UPR resin although it is produced at higher temperature. Identical findings have been reported by Monti et al. [32] on studying the effect of the addition of carbon nanofiber on the curing kinetics of UPR. The occurring of the post-curing of the UPR at higher temperature was explained considering that the free radicals generated by the initiator cannot react because they are trapped between the nanofibers. Chieruzzi et al. [33] also concluded that the addition of clay nanoparticles slowed down the curing and affected the final crosslinking rate of the UPR. 
The DSC thermograms of the UPR/PET fiber composites corresponding to the second heating run are represented in Figure $7 \mathrm{~b}$. They do not show any exothermic peak due to the complete cure of the matrix and display glass transition temperature only (Table 1). In the composite with 5 phr PET fiber, the increase in the Tg of the composites is due to the strong interactions between the PET fiber and the UPR matrix, causing restrictions to the chains mobility. The addition of higher PET fiber loading causes an important disruption in the crosslinking process and induces some plasticizing effect which decreases the Tg of the composites.

The thermal stability of the composites should be changed by adding PET fibers and it was studied by TGA. Figure 8a shows the variation of the weight loss as a function of the temperature for the PET fiber, the neat UPR and the UPR/PET fiber composites. The thermal decomposition of the composites occurs in one step. The degradation parameters, including the temperatures at which the decomposition starts and finishes noted as $\operatorname{Td}_{0}$ and $\operatorname{Td}_{\mathrm{f}}$, respectively, the temperature at the maximum of thermal decomposition $\operatorname{Td}_{\max }$, the decomposition rate $\mathrm{Vd}$, and the residual mass $\mathrm{M}_{\mathrm{res}}$ at the end of the TGA experiment were calculated and they are reported in Table 2.

The TGA thermogram of the neat UPR composite shows that it is thermally stable up to temperatures below $236^{\circ} \mathrm{C}$ which marks the beginning of its thermal decomposition. The neat UPR composite degraded fully at $447{ }^{\circ} \mathrm{C}$ rending a residual mass of $3 \mathrm{wt} \%$ due to char formation (Figure $8 \mathrm{~b}$ ). The weight loss at the maximal thermal decomposition located at 367 ${ }^{\circ} \mathrm{C}$ is $56 \mathrm{wt} \%$, occurring at a maximum rate of $2.1 \% / \mathrm{min}$. On the other hand, the thermal stability of the PET fiber is better than that of the UPR composite, and its decomposition starts at $342{ }^{\circ} \mathrm{C}$ and finishes at $491^{\circ} \mathrm{C}$ with a residual mass of $15 \mathrm{wt} \%$. The maximum of the weight loss is produced at $418{ }^{\circ} \mathrm{C}$ and the mass loss is $58 \mathrm{wt} \%$. The maximum speed of the weight loss is $2.0 \% / \mathrm{min}$.

The UPR/PET fiber composites exhibit better thermal stability than that of the neat UPR composite. The addition of 5-8 phr of PET fibers causes a good thermal stability at lower temperature but increases the decomposition temperature of the composites; however, the addition of more than 8 phr of PET fibers decreases the thermal stability of the composites, irrespective of the added amount. In fact, the DTGA thermograms of Figure $8 \mathrm{~b}$ reveal that 
the temperature at the maximum of decomposition shifts towards higher values which are intermediate between those of the UPR resin and the PET fiber. The maximum degradation temperature of the composites increases as the amount of the PET fiber increases. At the same time, the rate of maximum weight loss of the composites decreases by adding PET fibers (Table 2), suggesting that it causes a slowdown in the thermal decomposition of the composites and a significant improvement of their thermal stability mainly when 5-8 phr of PET fibers are added.

The viscoelastic properties of the UPR/PET fiber composites were studied by DMTA. The variation of the storage modulus $\left(E^{\prime}\right)$ as a function of the temperature for the neat UPR and the UPR/PET fiber (2-3 mm) composites are shown in Figure 9a. At lower temperatures, the variation of the storage modulus as a function of the temperature exhibits the glassy region in which the storage modulus does not vary by increasing the temperature. At the glass transition region, the storage modulus decreases sharply; once the glass transition region is finished, the characteristic rubbery plateau can be noticed. The addition of the PET fiber increases the storage modulus of the composites in the glassy region, and, in general, the higher is the amount of PET fiber, the higher is the storage modulus of the composite. The glass transition is initiated earlier in the composites containing PET fibers, and an increase in the glass transition temperature is obtained by adding 5-8 phr PET fibers; when $18 \mathrm{phr}$ of PET fibers is added, the glass transition temperature is similar to that of the neat UPR composite. Therefore, the addition of 5-8 phr of PET fibers enhances the properties of the UPR composite because of the existence of good interfacial interactions. On the other hand, in the rubbery plateau, the higher storage modulus corresponds to the composite containing $5 \mathrm{phr}$ of PET fibers.

Figure $9 \mathrm{~b}$ shows the variation of the damping factor $(\tan \delta)$ as a function of the temperature for the neat UPR and the UPR/PET fiber $(2-3 \mathrm{~mm})$ composites. The maxima in these plots correspond to the structural relaxations in the composites. The main structural relaxation relates to the glass transition of the composites and its temperature corresponds to their $\mathrm{T}_{\mathrm{g}}$ values. The $\mathrm{T}_{\mathrm{g}}$ of the neat UPR composite appears at $91{ }^{\circ} \mathrm{C}$ and only one structural relaxation can be distinguished. The addition of 5-8 phr of PET fibers increases the Tg value of the composite to 102 and $97{ }^{\circ} \mathrm{C}$ respectively, and it can be attributed to the good dispersion and the strong matrix/PET fiber interactions that limit the molecular mobility of the UPR chains. 
A similar trend was reported by Chieruzzi et al. [33] on studying UPR/montmorillonite (MMT) nanocomposites and they attributed the increase of the $T_{g}$ to the good adhesion between the clay and the matrix. Román et al. [34] also obtained the same trend in epoxy/MMT nanocomposites and the increase of the $\mathrm{T}_{\mathrm{g}}$ was ascribed to higher crosslinking density. However, the addition of $18 \mathrm{phr}$ of PET fibers decreases the $\mathrm{T}_{\mathrm{g}}$ value $\left(88^{\circ} \mathrm{C}\right)$ with respect to the neat UPR composite. Figure $9 \mathrm{~b}$ shows that a second minor structural relaxation appears in all UPR/PET fiber composites at about $50{ }^{\circ} \mathrm{C}$ which becomes more evident by increasing the PET fiber content. This second structural relaxation can be attributed to the creation of new PET fiber/UPR interactions that seems to be responsible of the above mentioned plasticizing effect. In fact, when the fiber content in the composite increases to 18 phr, the decrease in the $\mathrm{Tg}$ of the main structural relaxation could be attributed to lower crosslinking density or a plasticizing effect caused by the incorporation of a high amount of PET fibers which are very flexible [35].

According to Pothan et al. [35], Morote-Martínez et al. [36] and Aziz et al. [37], the extent of the interactions between reinforcement and polymer matrix can be evaluated from the height at the maximum of the curves of the $\tan \delta$ variation as a function of the temperature. Furthermore, the width at the half-height of the $\tan \delta$ peak can be related to the volume of the interface [33]. The height at the maximum and the width at the half-height of the tan $\delta$ peak of the composites are given in Table 3. The height at the maximum of $\tan \delta$ of the UPR/PET fiber composites decreases with respect to that of the neat UPR composite, irrespective to the added amount of PET fibers, which supports the existence of strong interactions between the fibers and the polymer matrix and/or the increase in the degree of crosslinking of the UPR resin. Additionally, the tan $\delta$ peak becomes narrower when 5-8phr PET fibers are added to the UPR but it becomes wider when 18 phr PET fibers are added, indicating that a larger interface is obtained by adding important amount of PET fiber.

\subsection{Influence of the PET fiber length on the properties of the UPR/PET fiber composites}

The study of the influence of the PET fiber content on the properties of the UPR/PET fiber composites has shown that the best mechanical performance was obtained by adding 5-8 phr of PET fibers. For determining the influence of the PET fiber length on the properties of the composites, 5 and $8 \mathrm{phr}$ of PET fibers were added to the UPR resin and the mechanical properties were studied. Figure 10a shows the variation of the tensile strength of the composites as a function of the PET fiber length. An increase in tensile strength is obtained in 
the composites made with short PET fiber lengths, i.e. 2-3 $\mathrm{mm}$ and $5 \mathrm{~mm}$; however, the addition of longer fibers causes a decrease in the tensile strength of the composites to a level similar or even lower than in the neat UPR composite. A similar trend is obtained in the elongation-at-break (Figure 10b) as the addition of short PET fibers causes an increase in the elongation-at-break. Toughening is imparted to the UPR/PET fiber composites because both the tensile strength and the elongation-at-break increase by adding PET fibers shorter than 5 $\mathrm{mm}$. The fibers are well oriented in the liquid matrix when they are short, and the longer are the PET fibers, the more they are flexible and the probability of entanglement in the PUR resin is enhanced. On the other hand, during the mechanical tests the areas where the longer PET fibers are bent exhibit poor impregnation with the UPR resin, which causes defects unable to support the stresses transfer, thereby causing a decrease in the tensile stress and elongation-at-break. The chance of creating defects in the composites made with short fibers is lower because a better wettability of the PET fiber by the resin is expected, owing a better dispersion in the polymer matrix. In fact, it has also been reported [30] that long fibers may confer a plastic behavior to the composite which results in a decrease of the tensile strength, but the defects due to poor wetting of the fibers were responsible for the decrease in the elongation-at-break.

The effect of the PET fiber length on the resilience of the UPR/PET fiber composites is shown in Figure 11. Similarly to the variation in tensile strength, the composites made with PET fibers of a length lower than 5-10 mm show improved impact resistance or resilience. The entanglements in the PET fibers and their poor dispersion are favored in the longer fibers and induce insufficient impregnation resulting in micro voids formation. As a result, the resilience decreases because the stresses transfer from one phase to another is not adequately ensured. On the other hand, the low resilience of the composites made with longer PET fibers may be also ascribed to their plasticizing effect.

The PET fiber length also affects the viscoelastic properties of UPR/PET fiber composites. The variation of the storage modulus as a function of the temperature for the composites made 
with $8 \mathrm{phr}$ of PET fibers of different lengths is given in Figure 12a. The composite reinforced by PET fibers of 2-3 mm length shows higher storage modulus than the one made with longer PET fibers. This is particularly more noticeable in the glass transition region where the short PET fibers impart a significant strengthening to the composite which is reflected by a larger storage modulus than for the neat UPR composite and the composites reinforced with PET fibers 5 and $10 \mathrm{~mm}$ long. Furthermore, the storage moduli of these later composites are significantly lower than that of the unfilled composite. The reinforcing effect revealed by the short PET fibers can be ascribed to their good dispersion and adhesion to the matrix which enables an efficient stresses transfer in the composite. In opposite, the longer PET fibers tend to bend and tangle and their great flexibility could reduce the storage modulus of the composites.

Figure $12 \mathrm{~b}$ shows the variation of the loss factor $(\tan \delta)$ as a function of the temperature for the UPR/PET fiber composites made with fibers of different lengths. The $T_{g}$ values of the composites are affected by the length of the PET fibers. Addition of PET fibers of 2-3 mm length increases the $T_{g}$, reduces the value of the maximum in the tan $\delta$ peak and reduces the peak width at half height with respect to the neat UPR composite (Table 4), indicating the existence of strong fiber/polymer interfacial interactions and/or higher degree of crosslinking. The increase in the length of the PET fiber to $5 \mathrm{~mm}$ does not produce significant changes in the $T_{g}$ value or the maximum in the tan $\delta$ peak because the interfacial interactions are not significantly increased. Finally, the addition of the PET fiber with $10 \mathrm{~mm}$ causes an important decrease in the $T_{g}$ value of the composite but decreases noticeably the maximum in the $\tan \delta$ peak due to an important plasticizing effect of the fibers in the composites. In fact, Figure $12 \mathrm{~b}$ shows that a significant second structural relaxation at $55{ }^{\circ} \mathrm{C}$ appears in the composite, confirming the existence of two structures with different viscoelastic characteristics. In summary, the addition of the short PET fibers rends relatively more rigid composites in which strong interfacial interactions are produced; when the PET fibers are longer, the good affinity between the ester structures in the unsaturated polyester and the surface of the fiber promotes good adhesion to the matrix but the resulting composite is less stiff because of the flexibility of the PET fiber that induces a plasticizing effect. 


\section{CONCLUSIONS}

Because of the hollow cylindrical structure of the recycled PET fibers, light composites with good mechanical properties can be obtained. The mechanical properties of UPR/PET fiber composites were determined by the amount and the length of the PET fibers. The optimal tensile strength and impact strength were obtained by adding 5-8 phr of PET fibers with a length lower than $5 \mathrm{~mm}$, and toughness was imparted to the composites. The improved mechanical properties of the UPR/PET fiber composites were ascribed to the good adhesion resulting from the high affinity of the PET fiber surface for the UPR matrix which promoted the mechanism fracture by splitting of the fibers. The ductile fracture of the composites reinforced with 5-8 phr was confirmed by the deformation of the PET fiber and the roughness of the fractured surface.

Higher crosslinking density leading to an increase in the $T_{g}$ value of the composite and lower value of maximum in $\tan \delta$ were found in the UPR/PET composites made with low amount of PET fiber of short length. For higher PET fiber content and length, a decrease in the $T_{g}$ was produced because of the flexibility of the PET fibers that led to the creation of a second structural relaxation in DMTA experiments, and caused lower crosslinking density in the polymer matrix. On the other hand, the addition of PET fiber improved the thermal stability of the composites and displaced the post-curing of the composites to higher temperature reducing the enthalpy of post-curing when 5-8 phr PET fiber of short length was added.

This study demonstrated the usefulness of adding short recycled PET fibers as reinforcement in UPR composites. The use of the PET fiber avoided the needs of a surface treatment for improving the interfacial adhesion to the UPR matrix because of the chemical affinity between the two polyesters, i.e. the PET fiber and the unsaturated polyester resin. 


\section{REFERENCES}

1. M. Alexandre and P. Dubois, Mater. Sci. Eng., 28, 1 (2000).

2. L. Xu and J. Lee, Polymer, 45, 7325 (2004).

3. B. Mortaigne, S. Bourbigot, M.L. Bras, G. Cordellier, A. Baudry and J. Dufay, Polym. Degrad. Stab., 64, 443 (1999).

4. K. Johnson and L. Yang. In: J. Scheirs, T.E. Long, editor. Modern polyesters: chemistry and technology of polyesters and copolyesters. John Wiley \& Sons, Chichester, P.699-712. (2003).

5. G. Huang and H. Sun, Materials and Design, 28, 1647 (2007).

6. M. Sham, J. Kim and J. Wu, Polymer Composites, 5, 165 (1997).

7. H. Mahiou and B. Akou, Composites : Part A, 29, 1035 (1998).

8. R. Graf, J. Koenig and H. Ishida, J. Adhes., 16, 97 (1983).

9. Y. Suzuki, Z. Maekawa, H. Hamada, A. Yokoyama and T. Sugihara, J. Mater. Sci., 28, 1725 (1993).

10. A. Gren, R. Joffe, L. Berglund and E. Mader, Composites: Part A, 30, 1009 (1999).

11. C. Varga, N. Miskolczi, L. Bartha and G. Lipoczi, Materials and Design, 31,185 (2010).

12. K. Rot, M. Huskic, M. Makarovic, T. L. Mlakar and M. Zígon, Composites: Part A, 32, $511(2001)$.

13. C. Soutis, Mater. Sci. Eng.: A., 412, 171 (2005).

14. A. DiBenedetto, Mater. Sci. Eng.: A, 302, 74 (2001).

15. S. Park and J. Jin, J. Colloid. Interface Sci., 242, 174 (2001).

16. H. Abdul Khalil and H. Ismail, Polym. Test., 20, 65 (2001).

17. L. Drzal and M. Madhukar, J. Mater. Sci., 28, 569 (1993).

18. E. Fitzer and R. Weiss, Carbon, 25, 455 (1987). 
19. C. Baillie, J. Watts, J. Castle and M. Bader, Compos. Sci. Technol., 48, 97 (1993).

20. C. Baley, F. Busnel, Y. Grohens and O. Sire, Composites: Part A, 37, 1626 (2006).

21. Y. Li, Y. Mai and L. Ye, Compos. Sci. Technol., 60, 2037 (2000).

22. P. Sreekumar, K. Joseph, G. Unnikrishnan and S. Thomas, Compos. Sci. Technol., 67, 453 (2007).

23. E. Bisanda, Appl. Compos. Mater., 7, 331 (2000).

24. A. Bismarck, A. Mohanty, I. A. Askargorta, S. Czapla, M. Misra, G. Hinrichsen and J. Springer. Green Chem., 3, 100 (2001).

25. A. Mohanty, M. Khan and G. Hinrichsen, Compos. Sci. Technol., 60, 1115 (2000).

26. C. Hill and H. Abdul Khalil, J. Appl. Polym. Sci., 78, 1685 (2000).

27. L. Pothan, J. George and S. Thomas, Composites Inter., 9, 335 (2002).

28. J. Rout, M. Mishra, S. Tripathy, S. Nayak and A. Mohanty, Compos. Sci. Technol., 61, 1303 (2001).

29. M. Rokbi, H. Osmani, A. Imad and N. Benseddiq, Procedia Engineering, 10, 2092 (2011).

30. P. H. Franco and A.V. González, Composites: Part B, 36, 597 (2005).

31. X. Ramis and J.M. Salla, J. Polym. Sci. B Polym. Phys., 35, 371 (1997).

32. M. Monti, D. Puglia, M. Natali, L. Torre and J.M. Kenny, Compos. Sci. Technol., 71, 1507 (2011).

33. M. Chieruzzi, A. Miliozzi and J. Kenny, Composites: Part A, 45, 44 (2013).

34. F. Román, Y. Calventus, P. Colomer and J.M. Hutchinson, Thermochimica Acta, 54, 76 (2012).

35. L.A. Pothan, Z. Ommen and S. Thomas, Compos. Sci. Technol., 63, 283 (2003).

36. V. Morote-Martínez, R. Torregrosa-Coque and J.M. Martín-Martínez, Int. J. Adhes. Adhes., 31,154 (2011). 
37. S.H. Aziz, P.M. Ansell, J.S. Clarke, and R.S. Panteny, Compos. Sci. Technol., 65, 525 (2005).

1

2

3

4

5

6

10

11

12

13

14

15

16

17

18

19

20

21

22

23

24

25

26

27

28

29

30

31

32

33

34

35

36

37

38

39

40

41

42

43

44

45

46

47

48

49

50

51

52

53

54

55

56

57

58

59

60
19

John Wiley \& Sons 
FIG. 1. Variation of the density of UPR/PET fiber composites as a function of the PET fiber content. $296 \times 209 \mathrm{~mm}$ (300 x 300 DPI) 
FIG. 2. SEM micrograph of the PET fibers. $338 \times 254 \mathrm{~mm}(96 \times 96 \mathrm{DPI})$ 

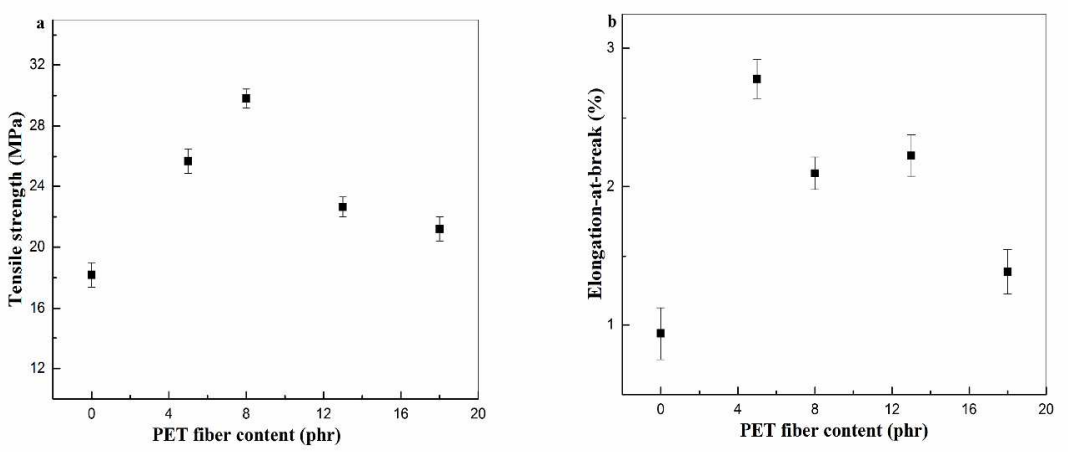

FIG. 3. Variation of the tensile strength (a) and the elongation-at-break (b) of the UPR/PET fiber composites as a function of the PET fiber content. $689 \times 368 \mathrm{~mm}(300 \times 300 \mathrm{DPI})$ 
1

2

3

4

5

6

7

8

9

10

11

12

13

14

15

16

17

18

19

20

21

22

23

24

25

26

27

28

29

30

31

32

33

34

35

36

37

38

39

40

41

42

43

44

45

46

47

48

49

50

51

52

53

54

55

56

57

58

59

60

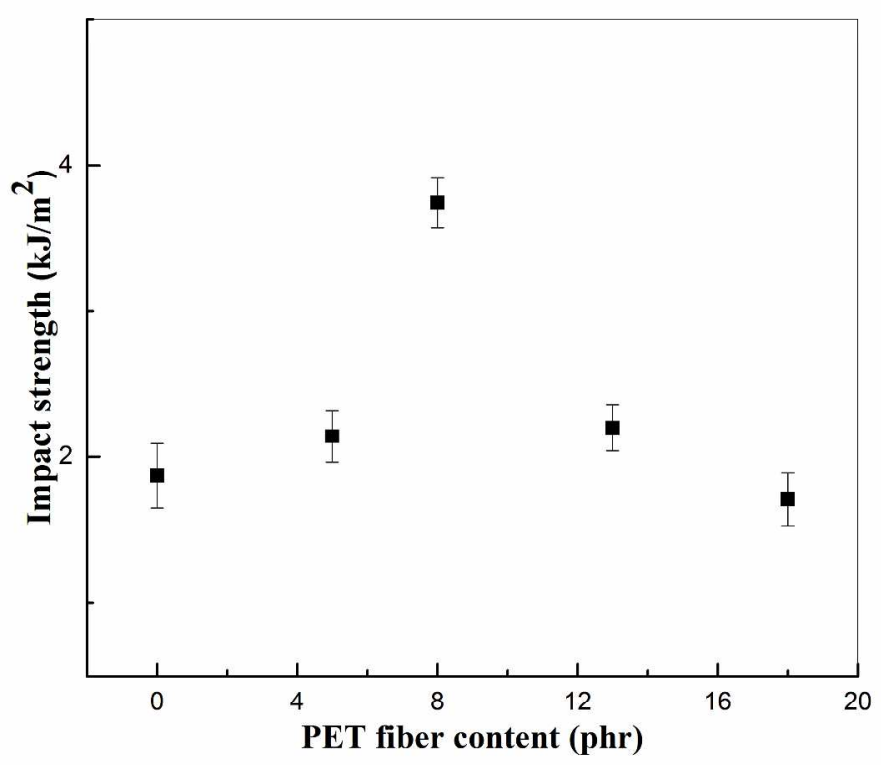

FIG. 4. Variation of the Izod impact strength of the UPR/PET fiber composites as a function of the PET fiber content.

$296 \times 209 \mathrm{~mm}(300 \times 300$ DPI $)$ 
FIG. 5. SEM micrographs of the UPR/PET fiber composites (a) UPR/5 phr PET fiber , (b) UPR/ 8 phr PET fiber and (c) UPR/18 phr PET fiber.

$338 \times 254 \mathrm{~mm}(96 \times 96 \mathrm{DPI})$ 
1

2

3

4

5

6

7

8

9

10

11

12

13

14

15

16

17

18

19

20

21

22

23

24

25

26

27

28

29

30

31

32

33

34

35

36

37

38

39

40

41

42

43

44

45

46

47

48

49

50

51

52

53

54

55

56

57

58

59

60
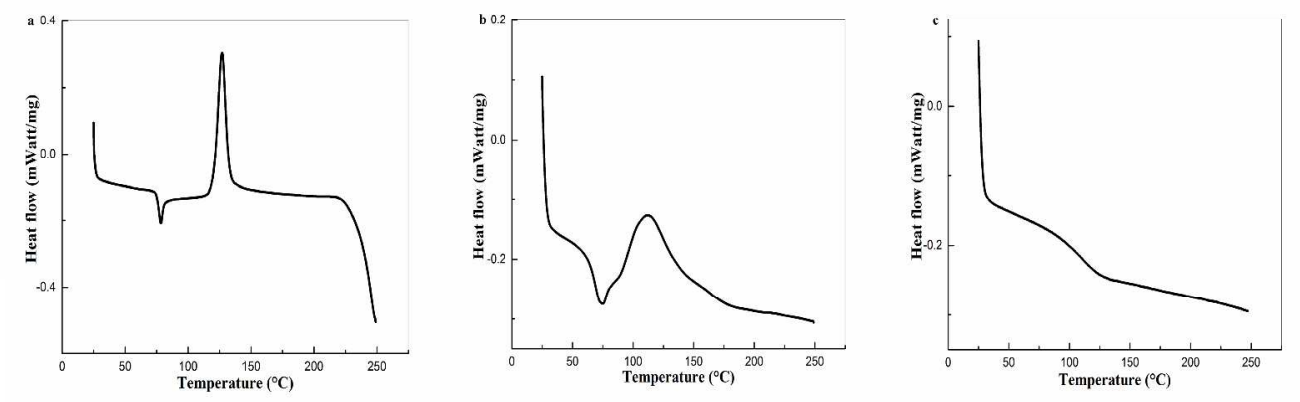

FIG. 6. DSC thermograms corresponding to the PET fiber (a), the first heating run (b) and the second heating run $(\mathrm{c})$ of the neat UPR

$687 \times 367 \mathrm{~mm}(300 \times 300$ DPI) 

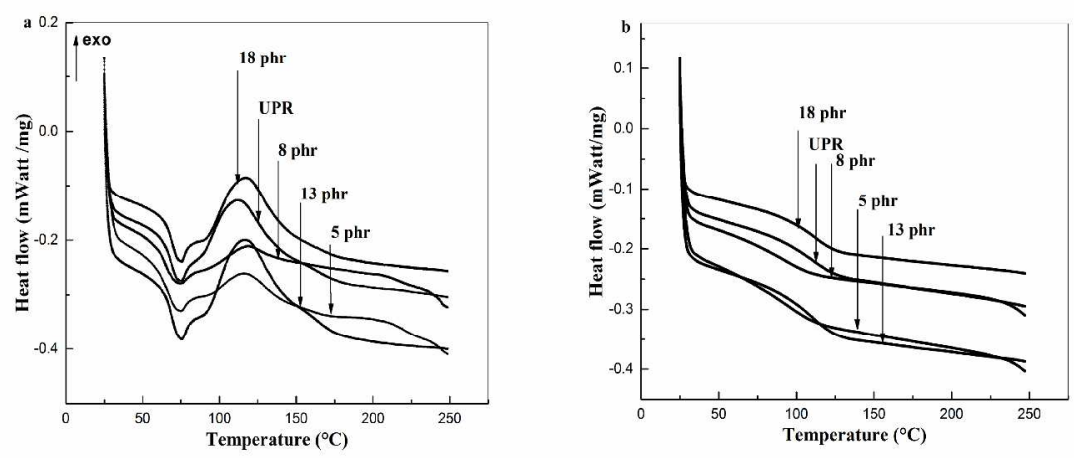

FIG. 7. DSC thermograms corresponding to the first heating run (a) and the second heating run (b) of the neat UPR and UPR/PET fiber composites. $689 \times 369 \mathrm{~mm}(300 \times 300 \mathrm{DPI})$ 
1

2

3

4

5

6

7

8

9

10

11

12

13

14

15

16

17

18

19

20

21

22

23

24

25

26

27

28

29

30

31

32

33

34

35

36

37

38

39

40

41

42

43

44

45

46

47

48

49

50

51

52

53

54

55

56

57

58

59

60
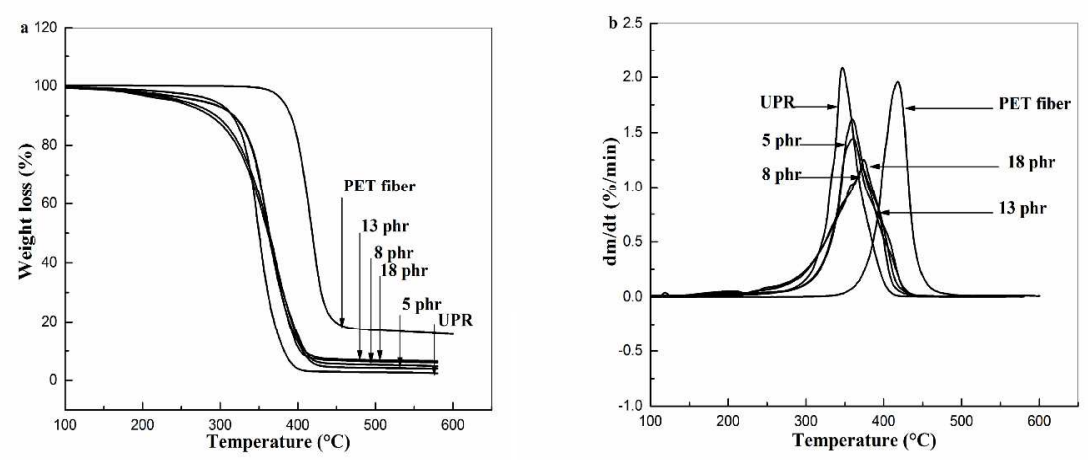

FIG. 8. Variation of the weight loss (a) and the weight loss derivative (b) as a function of the temperature for the UPR/PET fiber composites with different PET fiber content. $689 \times 367 \mathrm{~mm}$ (300 x 300 DPI) 

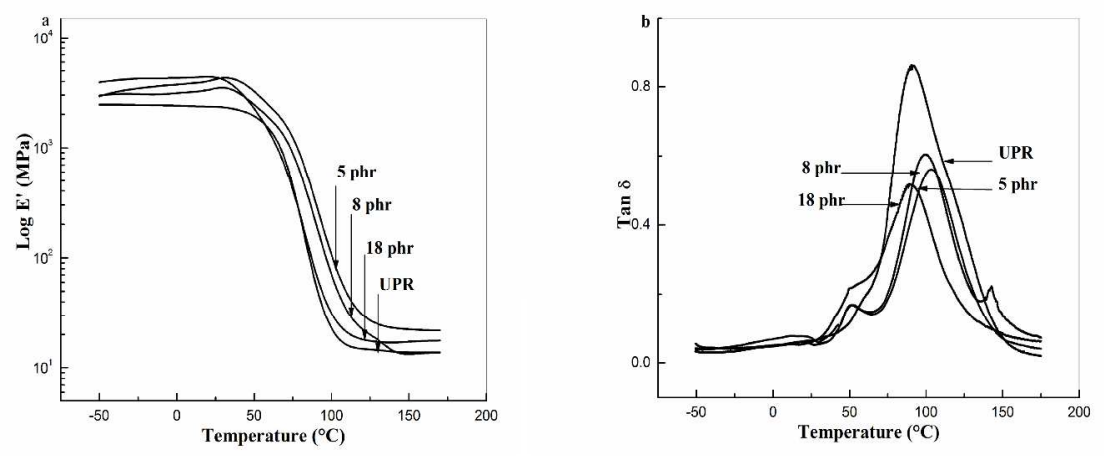

FIG. 9. Variation of the storage modulus (a) and $\tan \delta(b)$ as a function of temperature for the neat UPR and UPR/PET fiber (2-3 $\mathrm{mm})$ composites. $689 \times 366 \mathrm{~mm}(300 \times 300 \mathrm{DPI})$ 
1

2

3

4

5

6

7

8

9

10

11

12

13

14

15

16

17

18

19

20

21

22

23

24

25

26

27

28

29

30

31

32

33

34

35

36

37

38

39

40

41

42

43

44

45

46

47

48

49

50

51

52

53

54

55

56

57

58

59

60
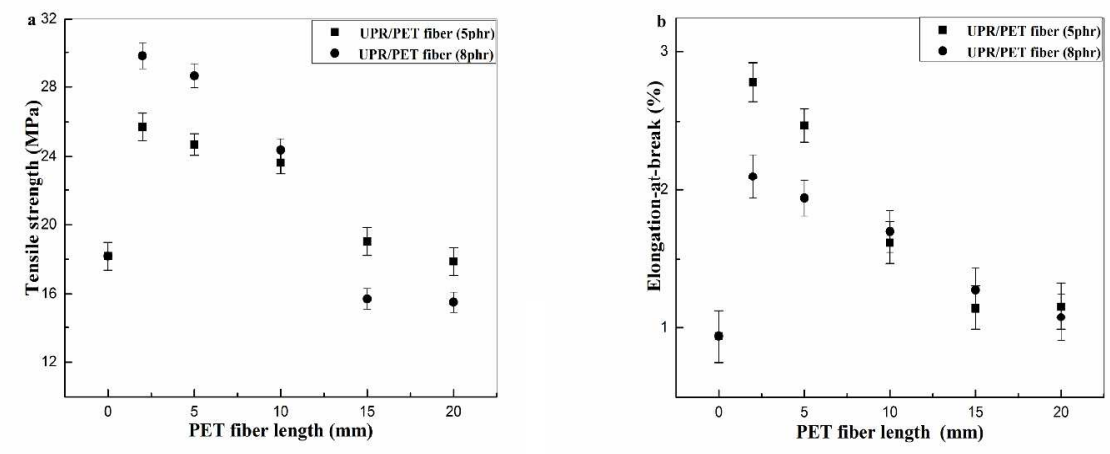

FIG. 10. Variation of the tensile strength (a) and the elongation-at-break (b) of the UPR/PET fiber composites as a function of the PET fiber length. $688 \times 367 \mathrm{~mm}(300 \times 300 \mathrm{DPI})$ 
FIG. 11. Variation of the Izod impact strength of the UPR/PET fiber composites as a function of the PET fiber length. $296 \times 209 \mathrm{~mm}(300 \times 300$ DPI $)$ 
1

2

3

4

5

6

7

8

9

10

11

12

13

14

15

16

17

18

19

20

21

22

23

24

25

26

27

28

29

30

31

32

33

34

35

36

37

38

39

40

41

42

43

44

45

46

47

48

49

50

51

52

53

54

55

56

57

58

59

60
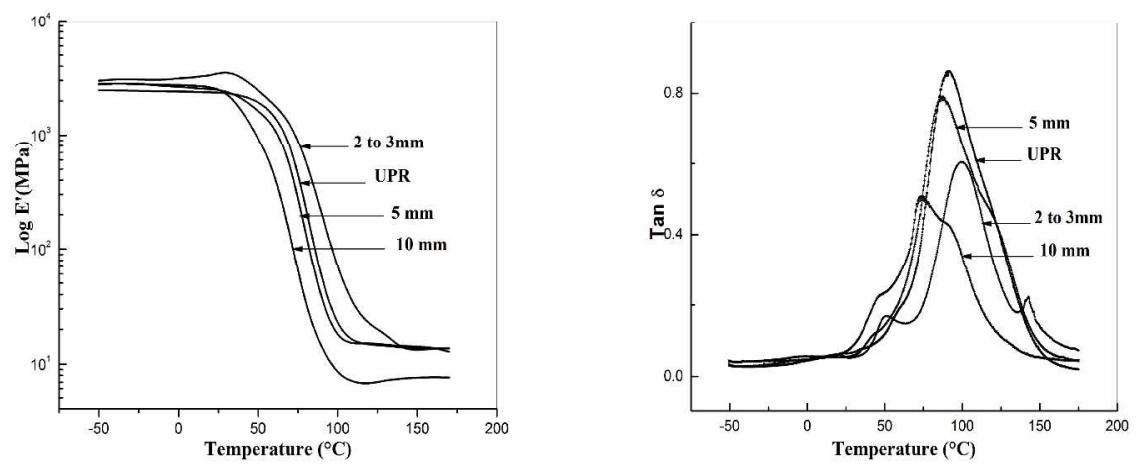

FIG. 12. Variation of the storage modulus (a) and $\tan \delta(b)$ as a function of temperature for the UPR/PET fiber composites with PET fiber content of $8 \mathrm{phr}$ and different fiber lengths.

$684 \times 382 \mathrm{~mm}(300 \times 300 \mathrm{DPI})$ 
TABLE 1. Post-curing temperature and enthalpy and glass transition temperature values of the neat UPR and UPR/PET fiber composites.

\begin{tabular}{lccc}
\hline \multicolumn{1}{c}{ Samples } & $\mathbf{T}_{\text {post-cure }}{ }^{\mathbf{a}}\left({ }^{\circ} \mathbf{C}\right)$ & $\Delta \mathbf{H}_{\text {post-cure }}{ }^{\mathbf{a}}(\mathbf{J} / \mathbf{g})$ & $\mathbf{T g}^{\mathbf{b}}\left({ }^{\circ} \mathbf{C}\right)$ \\
\hline UPR & 112 & 21 & 91 \\
UPR / PET fiber (5 phr) & 118 & 7 & 96 \\
UPR/ PET fiber (8 $\mathbf{~ h r})$ & 118 & 4 & 92 \\
UPR/ PET fiber (13 phr) & 116 & 27 & 82 \\
UPR/ PET fiber (18 $\mathbf{~ h r ) ~}$ & 116 & 24 & 86 \\
\hline
\end{tabular}

a determined from the first heating run

$\mathrm{b}$ determined from the second heating run 
1

2

3

4

5

6

7

8

9

10

11

12

13

14

15

16

17

18

19

20

21

22

23

24

25

26

27

28

29

30

31

32

33

34

35

36

37

38

39

40

41

42

43

44

45

46

47

48

49

50

51

52

53

54

55

56

57

58

59

60

TABLE 2. Values of the parameters of thermal decomposition of neat UPR, PET fiber and UPR/PET fiber composites.

\begin{tabular}{lcccccc}
\hline & & & \multicolumn{5}{c}{ UPR/PET fiber } \\
\cline { 5 - 7 } Parameter & PET fiber & $\mathbf{U P R}$ & $\mathbf{5 p h r}$ & $\mathbf{8 p h r}$ & $\mathbf{1 3 p h r}$ & $\mathbf{1 8 p h r}$ \\
$\mathbf{T}_{\mathbf{d} 0}\left({ }^{\circ} \mathbf{C}\right)$ & 342 & 236 & 300 & 297 & 277 & 272 \\
$\mathbf{T}_{\mathbf{d f}}\left({ }^{\circ} \mathbf{C}\right)$ & 491 & 447 & 478 & 465 & 474 & 457 \\
$\mathbf{T}_{\text {dmax }}\left({ }^{\circ} \mathbf{C}\right)$ & 418 & 367 & 380 & 380 & 393 & 395 \\
$\mathbf{V}_{\mathbf{d}}(\% / \mathbf{m i n})$ & 2.0 & 2.1 & 1.6 & 1.4 & 1.2 & 1.3 \\
Weight loss at $_{\mathbf{m}} \mathbf{T}_{\text {dmax }}(\%)$ & 58 & 56 & 57 & 55 & 39 & 36 \\
$\mathbf{m}_{\text {res }}(\%)$ & 15 & 3 & 3 & 3 & 6 & 5 \\
\hline
\end{tabular}


TABLE 3. Some parameters obtained from the DMTA plots of the neat UPR and UPR/PET fiber (2-3 $\mathrm{mm})$ composites.

\begin{tabular}{lccc}
\hline \multicolumn{1}{c}{ Samples } & $\mathbf{T g}\left({ }^{\circ} \mathbf{C}\right)$ & Max tan $\delta$ & Peak width at half height (cm) \\
\hline UPR & 91 & 0.86 & 1.40 \\
UPR/PET fiber (5phr) & 102 & 0.56 & 1.20 \\
UPR/PET fiber (8phr) & 97 & 0.60 & 1.30 \\
UPR/PET fiber (18phr) & 88 & 0.52 & 1.50 \\
\hline
\end{tabular}


1

2

3

4

5

6

7

8

9

10

11

12

13

14

15

16

17

18

19

20

21

22

23

24

25

26

27

28

29

30

31

32

33

34

35

36

37

38

39

40

41

42

43

44

45

46

47

48

49

50

51

52

53

54

55

56

57

58

59

60

TABLE 4. Some parameters obtained from the DMTA plots of the neat UPR and the UPR/PET fiber containing 8phr PET fibers with different lengths.

\begin{tabular}{lccc}
\hline Samples & Tg $\left({ }^{\circ} \mathbf{C}\right)$ & Max tan $\boldsymbol{\delta}$ & Peak width at half height $(\mathbf{c m})$ \\
\hline UPR & 91 & 0.86 & 1.40 \\
UPR/PET fiber (2-3 $\mathbf{~ m})$ & 97 & 0.60 & 1.30 \\
UPR/PET fiber $(\mathbf{5} \mathbf{~ m m})$ & 87 & 0.78 & 1.60 \\
UPR/PET fiber $(\mathbf{1 0} \mathbf{~ m m})$ & 74 & 0.50 & 1.50 \\
\hline
\end{tabular}

